OPEN ACCESS

Edited by:

Ran Wang,

Institute of Plant Protection and Environmental Protection, Beijing Academy of Agricultural and Forestry

Sciences, China

Reviewed by:

Yuichiro Suzuki,

Wellesley College, United States

Qi Su,

Yangtze University, China Honglin Feng,

Boyce Thompson Institute,

United States

*Correspondence:

Lin Jin

jinlin@njau.edu.cn

Specialty section

This article was submitted to Invertebrate Physiology, a section of the journal

Frontiers in Physiology

Received: 06 December 2021

Accepted: 10 January 2022

Published: 24 February 2022

Citation:

Ze L-J, Jin L and Li G-Q (2022) Silencing of Adc and Ebony Causes Abnormal Darkening of Cuticle in Henosepilachna vigintioctopunctata.

Front. Physiol. 13:829675. doi: 10.3389/fphys.2022.829675

\section{Silencing of Adc and Ebony Causes Abnormal Darkening of Cuticle in Henosepilachna vigintioctopunctata}

\author{
Long-Ji Ze, Lin Jin* and Guo-Qing Li
}

Education Ministry Key Laboratory of Integrated Management of Crop Diseases and Pests/State \& Local Joint Engineering Research Center of Green Pesticide Invention and Application, Department of Entomology, College of Plant Protection, Nanjing Agricultural University, Nanjing, China

$\mathrm{N}$ - $\beta$-alanyldopamine (NBAD) is a precursor of $\mathrm{N}$-acylquinone sclerotin utilized for crosslinking between cuticular proteins for cuticle during insect molting. The importance of NBAD in cuticle tanning has not been well compared among different developing stages of insects. Henosepilachna vigintioctopunctata, a typical polyphagous pest feeding on a large number of Solanaceae and Cucurbitaceae plants in Asian countries, displays diverse cuticle pigmentation patterns among developing stages and body regions. Here, we found that the expression of three genes (Hvadc, Hvebony, and Hvtan) involved in NBAD biosynthesis peaked in the 4-day-old pupae or 0-day-old adults of $H$. vigintioctopunctata. At the first, second, third, and fourth larval instar and pupal stage, their transcript levels were high just before and/or right after the molting. Moreover, they were more abundantly transcribed at the larval heads than in the bodies. RNA interference (RNAi) of either Hvadc or Hvebony at the third instar larvae selectively deepened the color of the larval head capsules, antennae, mouthpart, scoli, strumae, and legs; and depletion of the two genes blackened the pupal head capsules, antennae, mouthpart, and legs. However, the knockdown of either Hvadc or Hvebony darkened the whole bodies of the adults. Conversely, RNAi of Hvtan at the third instar stage had little influence on the pigmentation in the larvae, pupae, and adults. These findings demonstrated that Adc and Ebony are important in cuticle pigmentation of $H$. vigintioctopunctata and suggested that larger quantities of NBAD were present in adults and play more important roles in pigmentation than larvae/pupae.

Keywords: N- $\beta$-alanyldopamine, biosynthesis, adult cuticle, pigmentation, Henosepilachna vigintioctopunctata

\section{INTRODUCTION}

In insects, the cuticle provides protection against physical injury and water loss, rigidness for muscle attachment and mechanical support, and flexibility in intersegmental and joint areas for mobility. During growth and metamorphosis, insects need to regularly shed off old exoskeletons and synthesize new cuticles to fit the continuously increasing body sizes and the stage-specific body shapes. The newly formed cuticle, mainly composed of cuticular proteins, chitin, and sclerotized reagents, needs to be tanned, a process involved in melanization and sclerotization (Sugumaran and Barek, 2016). Moreover, melanization and sclerotization of insect cuticles also 
determine pigmentation and play an important role in ecological adaption, such as in escaping predation, mimicry, sexual selection, signaling, and thermoregulation (Wright, 1987; True, 2003; Wittkopp and Beldade, 2009; van't Hof et al., 2011).

From a biochemical perspective, melanization and sclerotization of insect cuticles result from a combination of dark black and brown melanin and light yellow and colorless sclerotins (Wright, 1987; True, 2003; Wittkopp et al., 2003; Wittkopp and Beldade, 2009; Zhan et al., 2010). These melanizing and sclerotized reagents are produced from tyrosine. The tyrosine hydroxylase (TH) converts tyrosine to dopa. Subsequently, the dopa decarboxylase (DDC) converts dopa into dopamine. Dopa and dopamine are further converted to corresponding melanins (Andersen, 2010). The production of sclerotins from dopamine has been clarified in Drosophila melanogaster (Phillips et al., 2005). There are four reaction steps: (1) N-acylation of dopamine with acetyl-CoA to $\mathrm{N}$-acetyldopamine (NADA). (2) Decarboxylation of aspartic acid to $\beta$-alanine by aspartate 1-decarboxylase (ADC, Black). (3) N-acylation of dopamine with $\beta$-alanine to produce $\mathrm{N}-\beta$-alanyldopamine (NBAD) by NBAD synthase (Ebony). This reaction is reversible, with the reverse reaction catalyzed by an NBAD hydrolase (Tan). (4) Oxidation of NADA and NBAD to NADA-quinone and NBAD-quinone, which are polymerized to form the corresponding $\mathrm{N}$-acylquinoid sclerotins (Phillips et al., 2005; Simon et al., 2009; Noh et al., 2016; Mun et al., 2020).

The importance of normal pigmentation of two enzymes, ADC and Ebony, has been confirmed (Mun et al., 2020). For $\mathrm{ADC}$, levels of $\beta$-alanine are reduced in the heads of $a d c$ mutants compared to wild type in D. melanogaster (Hodgetts and Choi, 1974; Phillips et al., 2005; Borycz et al., 2012; Ziegler et al., 2013), in black body color mutant Tribolium castaneum (Kramer et al., 1984) and the black pupal ( $b p$ ) mutant of Bombyx mori (Dai et al., 2015). Deficiency of $\beta$-alanine causes a lack of NBAD and leads to black body color (Kramer et al., 1984; Dai et al., 2015). Treatment with $\beta$-alanine can restore these mutants to wild-type phenotype (Jacobs, 1974; Kramer et al., 1984; Roseland et al., 1987; Wappner et al., 1996a,b; Phillips et al., 2005).

As for Ebony, the mutation in or knockdown of ebony increases black pigments in Dipteran insects D. melanogaster and Ceratitis capitata (Bridges and Morgan, 1923), Lepidopteran insects B. mori (Futahashi et al., 2008) and Spodoptera litura (Bi et al., 2019), Coleopteran insects Tenebrio molitor (Mun et al., 2020) and T. castaneum (Tomoyasu et al., 2009), and Hemipteran insect Oncopeltus fasciatus (Liu et al., 2016). Similarly, Tan has been recognized as an additional factor that promotes melanization in Heliconius (Ferguson et al., 2011). In D. melanogaster, loss of Tan causes a global reduction of melanin patterns (True et al., 2005; Jeong et al., 2008). However, the importance of NBAD in cuticle tanning has not been well compared among different developing stages of insects.

Henosepilachna vigintioctopunctata (Fabricius) (Coleoptera: Coccinellidae) is a typical polyphagous pest that feeds on a large number of Solanaceae and Cucurbitaceae plants in Asian countries (Zhang et al., 2018). The color markings in the beetle are distinctive and variable in different developing stages (Casari and Teixeira, 2015). This offers a very suitable model to test the pigmentation patterns. Here, using RNA interference (RNAi) technology, the three genes adc, ebony, or tan reported being involved in NBAD biosynthesis were knockdown at the third larval instar stage of $H$. vigintioctopunctata. The cuticle pigmentation patterns were detected and compared during larval-larval, larval-pupal, and pupal-adult molting. The results demonstrate that Adc and Ebony are important in cuticle pigmentation of $H$. vigintioctopunctata and suggest that larger quantities of NBAD were present in adults and play more important roles in pigmentation than larvae/pupae.

\section{MATERIALS AND METHODS}

\section{Insect}

Henosepilachna vigintioctopunctata adults were collected from Solanum melongena L. in Nanjing city, Jiangsu Province, China, in the summer of 2018. The beetles were routinely maintained in an insectary at $28 \pm 1{ }^{\circ} \mathrm{C}$ under a $14: 10 \mathrm{~h}$ light-dark photoperiod and $50-60 \%$ relative humidity using potato (Solanum tuberosum) foliage at the vegetative growth or young tuber stages to assure sufficient nutrition. Under this feeding protocol, the larvae progressed through four distinct instars, with approximate periods of the first, second, third, and fourth instar stages of 3 , 2, 2, and 3 days, respectively. Upon reaching full size, the fourth larval instars stopped feeding, fixed their abdomen ends to the substrate surface, and entered the prepupal stage. The prepupae spent approximately 2 days to pupate. The pupae lasted about 4 days and the adults emerged.

\section{Molecular Cloning}

TRIzol reagent (Invitrogen, New York, NY, United States) was used to extract the total RNA following the protocols of the manufacturer. The NanoDrop 2000 spectrophotometer (Thermo Fisher Scientific, New York, NY, United States) was applied to perform the RNA quantification. RNA purity was determined by assessing optical density (OD) absorbance ratios at OD260/280 and OD260/230. The integrity of RNA was analyzed via $1 \%$ agarose gel electrophoresis with ethidium bromide staining. Reverse transcription was performed using a PrimeScript ${ }^{\mathrm{TM}}$ RT reagent Kit with a gDNA Eraser (TaKaRa Biotechnology Corporation Ltd., Dalian, China). Briefly, the reaction was incubated at $37^{\circ} \mathrm{C}$ for $15 \mathrm{~min}$ and then $85^{\circ} \mathrm{C}$ for $5 \mathrm{~s}$. The synthesized cDNAs were preserved at $-20^{\circ} \mathrm{C}$ for further use.

The putative $a d c$, ebony, and tan genes were obtained from H. vigintioctopunctata transcriptome data (Zhang et al., 2018). The correctness of the sequences was substantiated by PCR using primers in Supplementary Table 1. The sequenced cDNAs were submitted to GenBank (accession numbers: adc, MW380963; ebony, MW380964; and tan, MW380968).

The protein sequences of ADC, Ebony, and Tan from other species were acquired from the NCBI. ${ }^{1}$ Phylogenetic analysis was conducted using MEGA-X software ${ }^{2}$ and the neighbor-joining method with 1,000 bootstrap replications.

\footnotetext{
${ }^{1}$ https://www.ncbi.nlm.nih.gov/

${ }^{2}$ https://sourceforge.net/projects/mega5/
} 


\section{Synthesis of dsRNA Molecules}

The cDNA fragments derived from adc, ebony, tan, and enhanced green fluorescent protein $(e g f p)$ were, respectively, amplified by $\mathrm{PCR}$ using specific primers (Supplementary Table 1) conjugated with the T7 RNA polymerase promoter. These targeted regions were further BLAST (BLASTN) searched against $H$. vigintioctopunctata transcriptome data (Zhang et al., 2018) to identify any possible off-target sequences that had an identical match of $20 \mathrm{bp}$ or more. The dsRNA was synthesized using the MEGAscript T7 High Yield Transcription Kit (Ambion, Austin, TX, United States) according to the instructions of the manufacturer. Subsequently, the synthesized dsRNA (at a concentration of 5-8 $\mu \mathrm{g} / \mu \mathrm{l}$ ) was determined by agarose gel electrophoresis and the NanoDrop 1,000 spectrophotometer (Thermo Fisher Scientific, Waltham, MA, United States) (data not shown) and kept at $-80^{\circ} \mathrm{C}$ until used in the subsequent experiment.

\section{Larval RNA Interference}

RNA interference of larvae was performed according to a previously described method (Xu et al., 2020; Ze et al., 2021). Briefly, an aliquot $(0.1 \mu \mathrm{l})$ of the solution including $300 \mathrm{ng}$ dsRNA was injected into the newly ecdysed third instar larvae. Blank and negative control larvae were injected with the same volume of PBS and dsegfp solutions, respectively. A group of 15 injected larvae was set as a replicate. Each dsRNA injection was repeated 8 times. Three replicates (each replicate contained at least six individuals) were sampled at 48 and $72 \mathrm{~h}$ after injection for qRT-PCR to test RNAi efficacy. Another two replicates were used to observe the phenotype.

Three biological independent experiments were carried out using the newly ecdysed third instar larvae and were planned to determine the RNAi effects of Hvadc, Hvebony, and Hvtan on the performances and cuticle tanning of the resultant larvae, pupae, and adults. Three treatments were set as follows: phosphatebuffered saline (PBS), $300 \mathrm{ng}$ dsegfp and $300 \mathrm{ng}$ dsadc; PBS, $300 \mathrm{ng}$ dsegfp and $300 \mathrm{ng}$ dsebony; or PBS, $300 \mathrm{ng}$ dsegfp and $300 \mathrm{ng}$ dstan.

\section{Real-Time Quantitative PCR}

For temporal expression analysis, cDNA templates were derived from 3-day-old eggs, the first, second, third, and fourth larval instar, the prepupae, pupae, and adults at an interval of 1 day (D0 indicates newly molted larvae, pupae, or newly emerged adults). For comparison of the expression levels in different portions, the heads and the remaining portions (bodies) were separately collected from 2-day-old third instar larvae, and 1-, 2-, and 3-day-old fourth instar larvae. Each sample contained 10 individuals and repeated three times. For analysis of the effects of treatments, total RNA was extracted from treated larvae. Each sample contained at least six individuals and repeated three times. The RNA was extracted using SV Total RNA Isolation System Kit (Promega, Madison, WI, United States). cDNA was prepared according to the instructions by using HiScript III RT SuperMix for qPCR (+gDNA wiper) kit (Vazyme, Nanjing, China). The qPCR performed on Applied Biosystems 7500 System (Life Technologies, Carlsbad, CA, United States) with ChamQ Universal SYBR qPCR Master Mix (Vazyme, Nanjing, China) according to the instructions of the manufacturer. Each $20 \mu \mathrm{l}$ reaction solution containing $10 \mu \mathrm{l}$ of $2 \times$ ChamQ Universal SYBR qPCR Master Mix, $0.4 \mu \mathrm{l}$ of each primer $(10 \mu \mathrm{M}), 1 \mu \mathrm{l}$ of cDNA template, and $8.2 \mu \mathrm{l}$ of nuclease-free water. The cycling parameters were: 1 cycle of $95^{\circ} \mathrm{C}$ for $3 \mathrm{~min} ; 40$ cycles of $95^{\circ} \mathrm{C}$ and $60^{\circ} \mathrm{C}$ for $10 \mathrm{~s}$ and $30 \mathrm{~s}$, respectively. Quantitative mRNA measurements were performed by qRT-PCR in technical triplicate, using two internal control genes (ribosomal protein S18, HvRPS18; ribosomal protein L13, HvRPL13; and the primers listed in Supplementary Table 1) according to the published results (Lü et al., 2018). An RT negative control (without reverse transcriptase) and a non-template negative control were included for each primer set to confirm the absence of genome DNA and to check for primer-dimer or contamination in the reactions, respectively.

According to a previously described method (Bustin et al., 2009), the generation of specific PCR products was confirmed by gel electrophoresis. The primer pair for each gene was tested with a 10-fold logarithmic dilution of a cDNA mixture to generate a linear standard curve [crossing point (CP) plotted vs. $\log$ of template concentration], which was used to calculate the primer pair efficiency. All primer pairs amplified a single PCR product with the expected sizes, showed a slope less than -3.0 , and exhibited efficiency values ranging from 2.5 to 2.6 . Data were analyzed by the $2^{-\Delta \Delta C T}$ method, using the geometric mean of the four internal control genes for normalization.

\section{Image Processing and Color Intensity Measurement}

Digital photographs of all the phenotypes were taken with Nikon SMZ 25 stereo microscopy (Nikon, Japan). Images of hindwings, dissected from 7-day-old adults whose third instar larvae were treated with dsRNA, were transferred to RGB stack images, and color intensity in equivalent regions was determined as mean grayscale values (average luminance) via ImageJ software. Under this measurement, the lower color intensity indicates the darker cuticle pigmentation (Noh et al., 2016). All the treated groups and the corresponding control groups were photographed under the same conditions.

\section{Data Analysis}

Statistical significance of differences in the mRNA expression or other biological experiments among PBS, dsegfp, and dsRNA treatment groups was determined by one-way ANOVA and Turkey's test in SPSS Statistics 20.0 software (at $P<0.05$ ). In the color intensity measurement of hindwings, the statistical significance of differences in mean gray values between the dsRNA-treated groups was assessed by Student's $t$-test. 


\section{RESULTS}

\section{Temporal Expression Pattern of Adc, Ebony, and Tan Transcript}

By mining of $H$. vigintioctopunctata transcriptome data and constructing the phylogenetic trees, Hvadc, Hvebony, and Hvtan were identified (Supplementary Figures 1-3).

To detect the expression patterns of Hvadc, Hvebony, and Hvtan during different developmental stages, qRT-PCR was performed. The results revealed that Hvadc, Hvebony, and Hvtan were detectable from the embryo (egg) to adults. They peaked in the 4-day-old pupae or 0-day-old adults. Moreover, at the first, second, third, and fourth larval instar and pupal stages, the transcription levels of Hvadc, Hvebony, and Hvtan were high just before and/or right after the molt, and were low at the intermediate stages (Figures 1A-C).
The expression levels of Hvadc, Hvebony, and Hvtan in larval heads were compared to those in the bodies of 2day-old third instar larvae, and 1-, 2-, and 3-day-old fourth instar larvae. Both Hvadc and Hvebony were abundantly transcribed in the larval heads of 2-day-old third instar larvae and 1-day-old four instar larvae (Figures 1D,E). Similarly, the levels of Hvtan in the head samples of 1- to 3-dayold fourth instar larvae were higher than those in the bodies (Figure 1F).

\section{RNA Interference of Adc Blackens the Larvae}

Three days after the introduction of dsadc into the newly ecdysed third instar larvae, the accumulated mRNA level of the target transcript was heavily suppressed (Figure 2A).
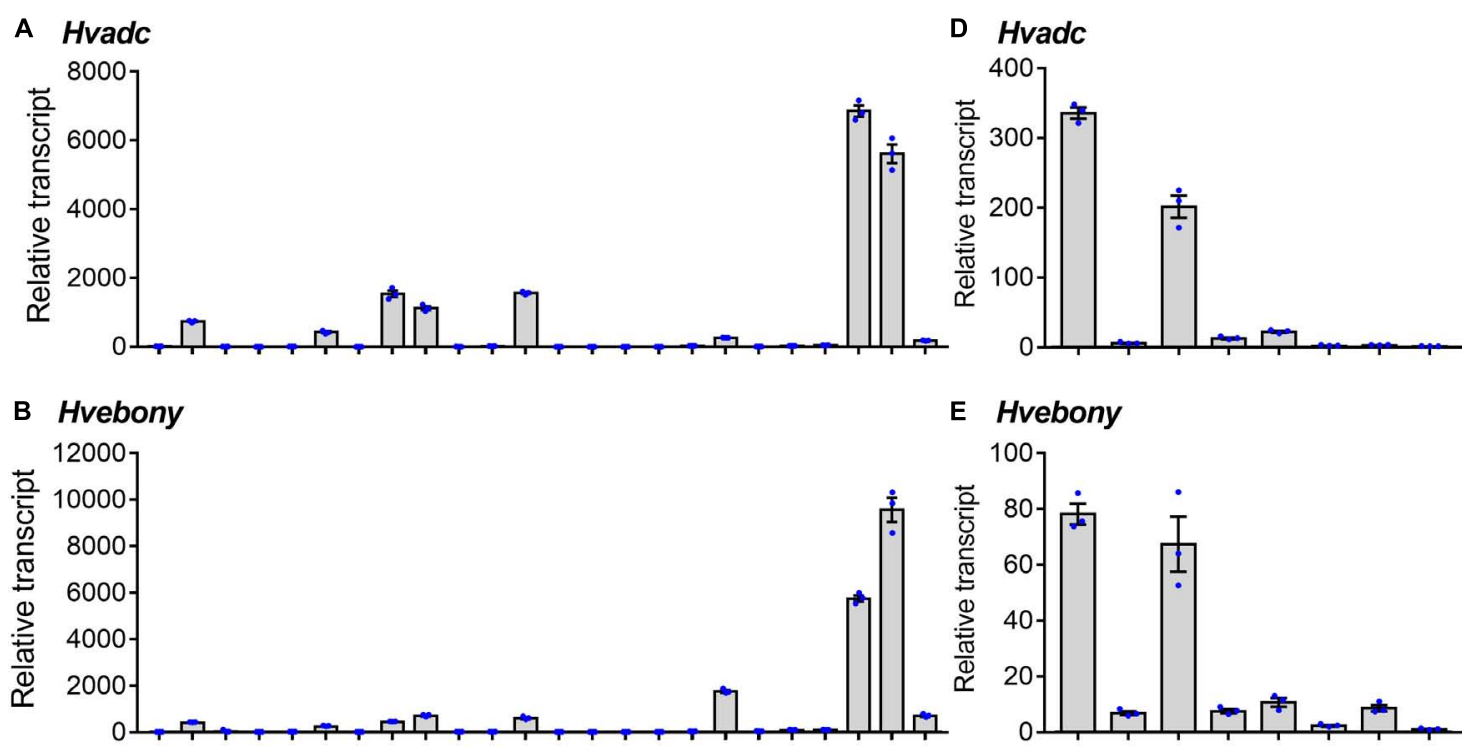

E Hvebony
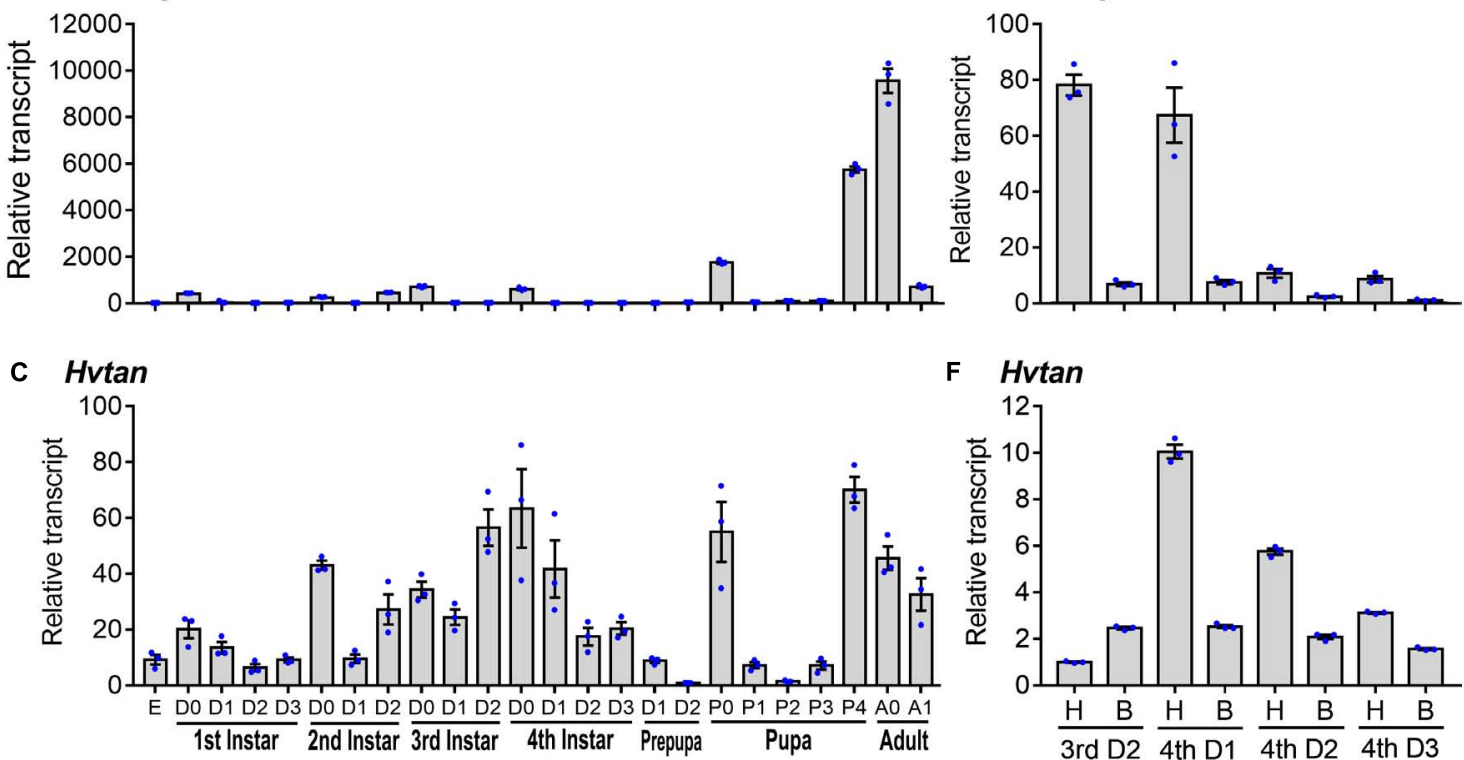

FIGURE 1 | Transcription patterns of adc, ebony, and tan genes in Henosepilachna vigintioctopunctata. For temporal expression analysis (A-C), RNA templates were derived from day 3 eggs, the larvae from the first through the fourth instar, prepupae, pupae, and adults (D0 indicated newly ecdysed larvae or pupae, or newly emerged adults). Each repeat included 20-30 individuals and there were three independent pools. For analysis of the expression levels in different regions (D-F), the heads and the remaining bodies were separately collected from the 2-day-old third instar larvae, and 1-, 2- and 3-day-old fourth instar larvae. A replicate included 10 individuals and each sample repeated 3 times. All the samples were measured in technical triplicate using qRT-PCR. The values were calculated using the $2-\Delta \Delta C T$ method. The lowest transcript level of each of the three mRNAs at a specific developing time is set as 1 . The relative transcripts are the ratios of copy numbers in different developing stages relative to larvae at the specific developing time. The genes for ribosomal protein S18 (HVRPS18) and ribosomal protein L13 (HVRPS13) were used as internal controls. The columns represent averages with vertical lines indicating $\pm \mathrm{SE}$. E, egg; $\mathrm{H}$, head; B, remaining body without head. 


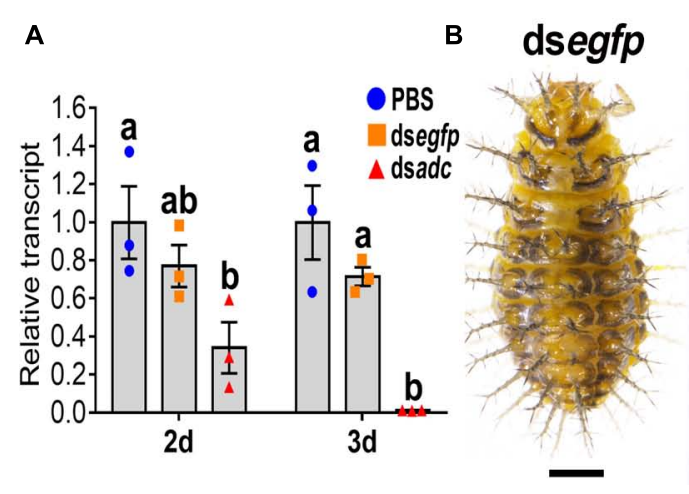

\section{E dsadc}

F dsegfp

G dsadc
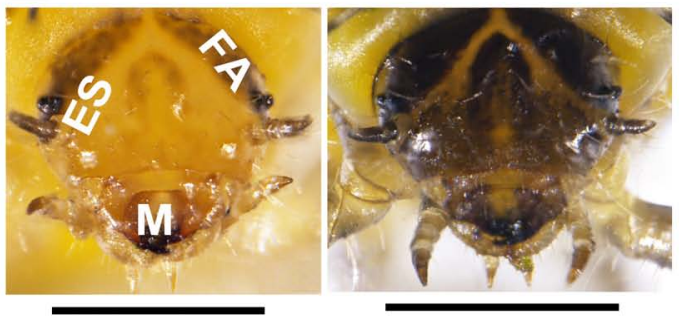

J dsegfp

K dsadc

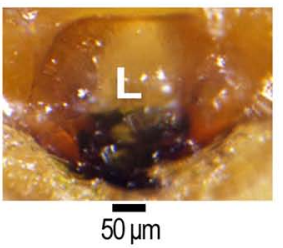

$\mathbf{N}$

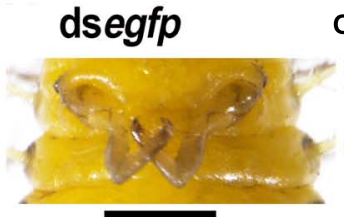

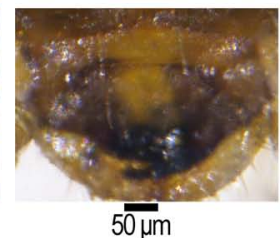

- dsadc

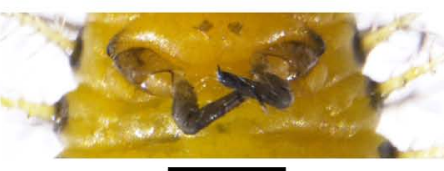

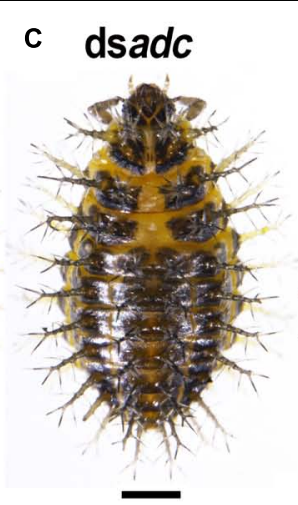

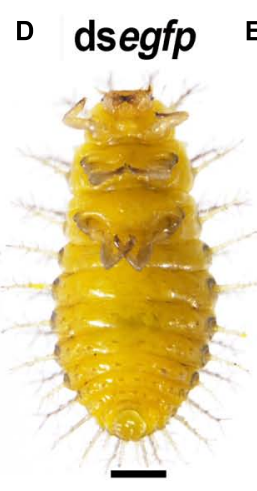

H dsegfp

I dsadc

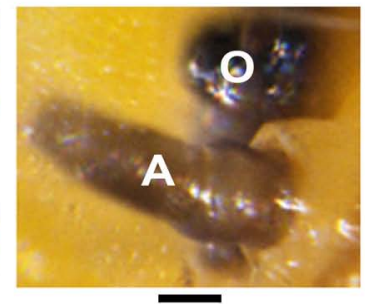

$\overline{50 \mu \mathrm{m}}$

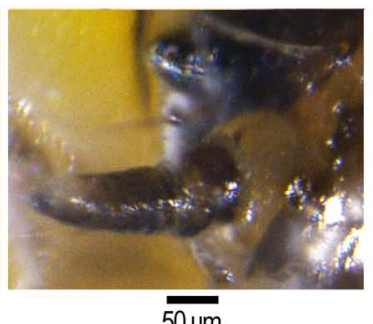

L dsegfp

M dsadc
P dsegfp

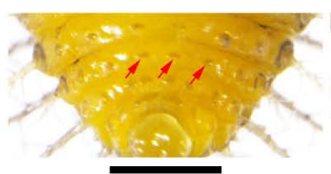

$\overline{50 \mu \mathrm{m}}$
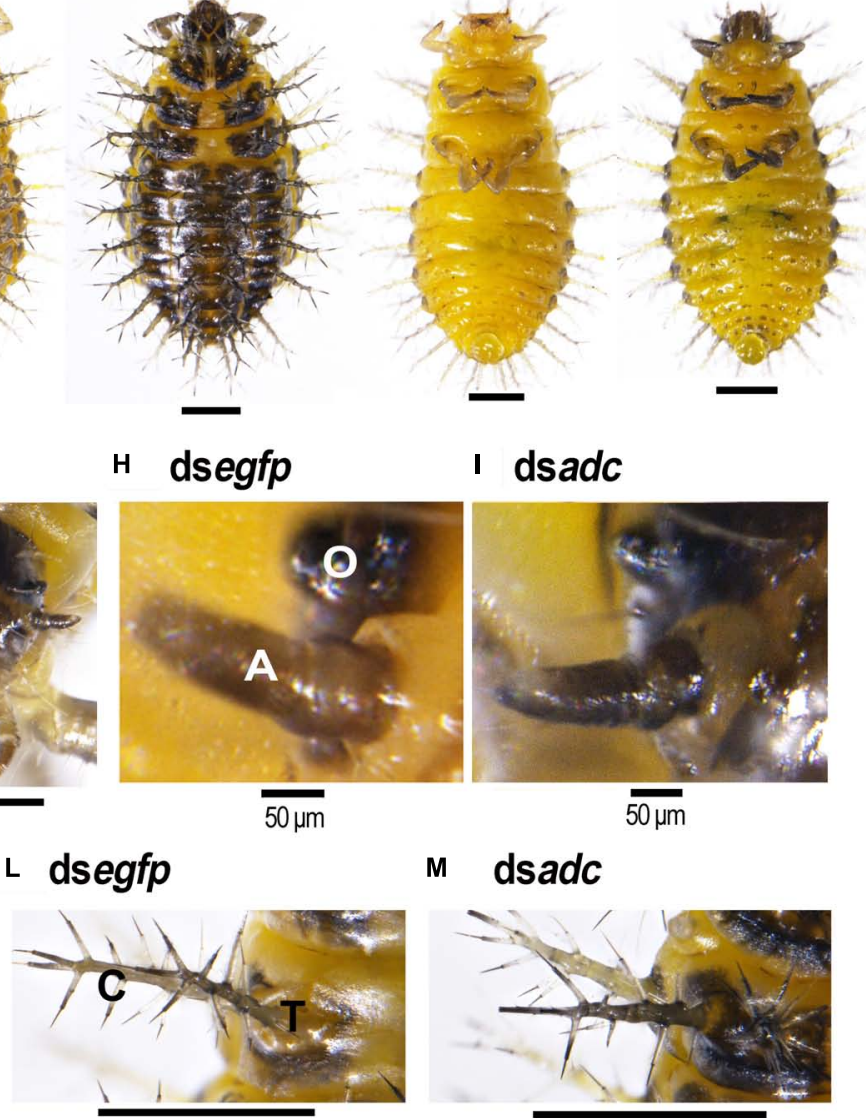

Q dsadc

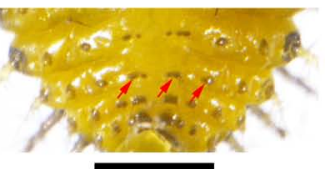

FIGURE 2 | RNA interference of Hvadc darkens larvae of $H$. vigintioctopunctata. The newly ecdysed third instar larvae were injected with an aliquot (0.1 $\mu$ l) of PBS, or solution including $300 \mathrm{ng}$ dsegfp or $300 \mathrm{ng}$ dsadc. The larvae were then transferred to potato foliage. Two days and three days after injection, the expression level of Hvadc was measured (A). Relative transcripts are the ratios of relative copy numbers in treated individuals to that in PBS-injected ones, which is set as 1 . Different letters indicate significant differences at $P$-value $<0.05$ using ANOVA with the Tukey-Kramer test. The dorsal and ventral views of dsegfp- (B,D) and dsadc- (C,E)

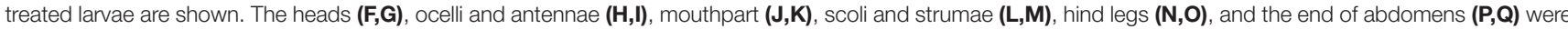
further amplified. ES, epicranial suture; FA, frontal arms; M, mouthpart; O, ocelli; A, antennae; C, scolus; T, struma. Red arrows point to mastoids on the abdomen.

All the controls (PBS- and dsegfp-introduced) and dsadctreated larvae normally molted to the fourth instar larvae. However, the color was darkened in the heads of the Hvadc RNAi larvae, compared with those of the PBS- and dsegfptreated ones (Figures 2B,D vs. C,E), especially the mouthparts (Figures 2F vs. G) and the patches around larval ocelli and the antennae (Figures $\mathbf{2} \mathbf{H}$ vs. I). In addition, a wide cream band called epicranial suture (ES) was narrowed, and the V-shaped frontal arms and the U-shaped patch on the head top were widened in the Hvadc RNAi larvae (Figures 2F vs. G).
In the mouthparts of the control fourth instar larvae (PBS- and dsegfp-injected), the median part of the labrum is cream, while the lateral and anterior parts are brownish narrow bands. Other appendages of the mouthparts, namely, mandibles, maxilla, and labium were black in color (Figures 2F,J). By contrast, in the Hvadc RNAi larvae, the color of all the pigmented regions was deepened and blackened (Figures 2F,J vs. G,K). Moreover, the scoli, strumae, and legs were all darker-colored in the Hvadc silenced larvae (Figures $2 \mathrm{~L}, \mathbf{N}$ vs. $\mathbf{M}, \mathbf{O}$ ). In addition, small mastoids queuing up on the abdomen were darker pigmented 


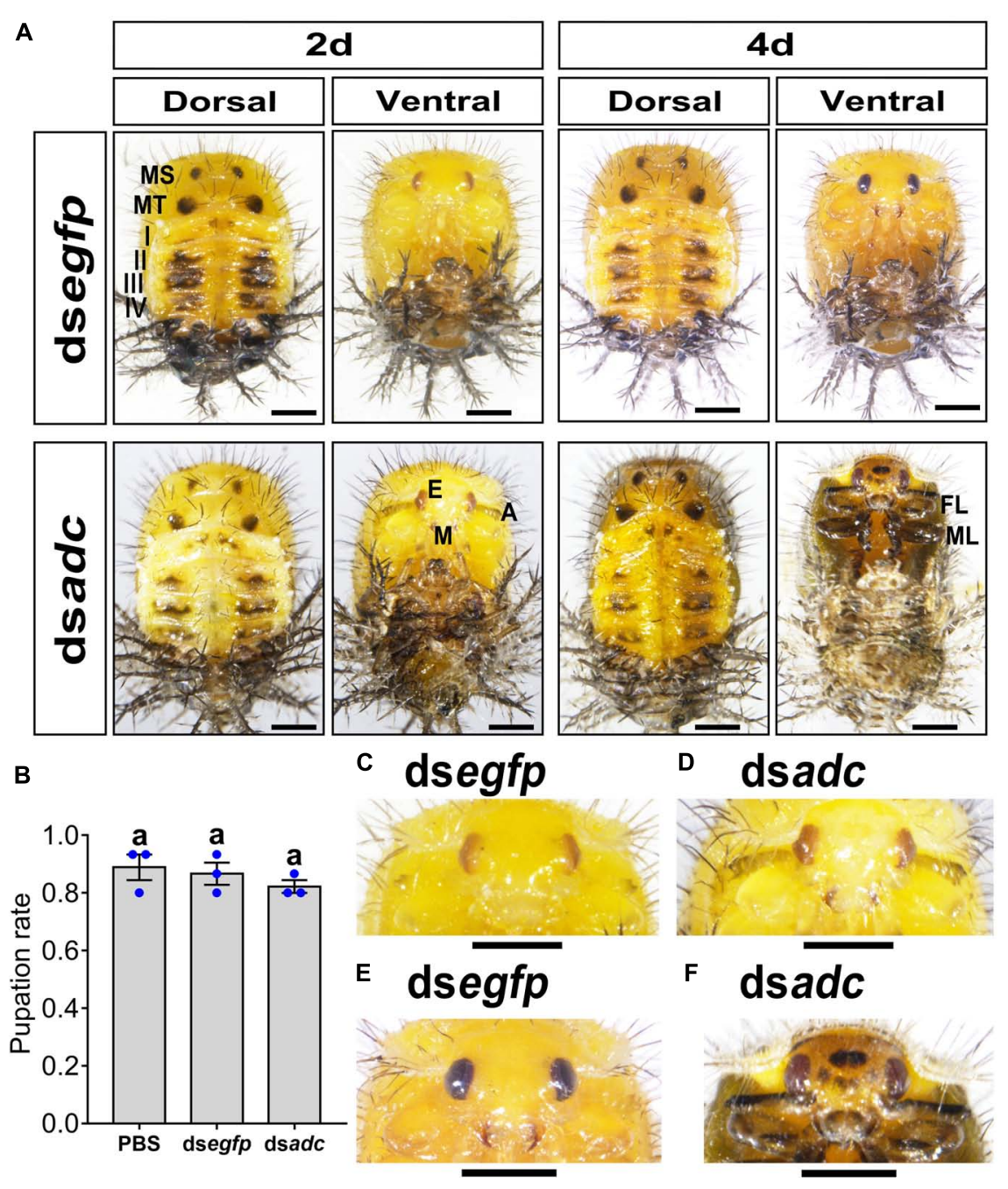

FIGURE 3 | Knockdown of Hvadc darkens pupa color in $\mathrm{H}$. vigintioctopunctata. The newly molted third instar larvae were injected with an aliquot (0.1 $\mu$ l) of PBS, or solution including $300 \mathrm{ng}$ dsegfp or $300 \mathrm{ng}$ dsadc. The larvae were then transferred to potato foliage. The pupation rate was recorded during a 3 -week trial period (B). The averages ( \pm SE) following different letters indicate significant differences at $P$-value $<0.05$ using ANOVA with the Tukey-Kramer test. The dorsal and ventral views of 2-day-old and 4-day-old pupae whose third instar larvae had been subjected to dsegfp and dsadc injection are shown (A). The heads of 2-day-old pupae (C,D) and 4-day-old pupae (E,F) were further amplified. MS, meso thorax; MT, meta thorax; I-IV, tergites I-IV; E, compound eye; A, antenna; M, mouthpart; FL, foreleg; ML, midleg. Scale bars: $1 \mathrm{~mm}$.

in dsadc-injected larvae, compared with that of dsegfp-injected larvae (Figures 2D,P vs. E,Q).

\section{Silencing Adc Affects Coloration of Pupae}

Knockdown of Hvadc did not affect the pupal morphology (Figure 3A) and the pupation rate (Figure 3B), but the coloration.

The integument of the 2-day-old pupae developed from the third instar larvae treated with dsegfp or dsadc were generally pale yellow, especially on the ventral one (Figure 3A, left panel). Then, the overall color shifted to dull yellow in the 4-day-old pupae (Figure 3A, right panel). From the dorsal views, the paired black markings in the meso and meta thoraxes, and the dark patches in tergite I-IV were similar between the control (dsegfp-treated) and the Hvadc RNAi pupae (Figure 3A).

However, the antennae and mouthparts of the 2-dayold Hvadc-silenced pupae were blackened (Figure 3D), while those parts in 2-day-old pupae treated with dsegfp exhibited no obvious pigmentation (Figure 3C). Four days after pupation, the mouthparts of control displayed rufous color, and the color of antennae and legs did not obviously change (Figure 3E). By contrast, the cuticle color between two compound eyes of the Hvadcsilenced pupae was obviously darker, and obvious central black patches and several small dark spots were present (Figures $3 \mathrm{E}$ vs. F). The antennae, mouthparts, and legs became darker (Figure 3F). 
A

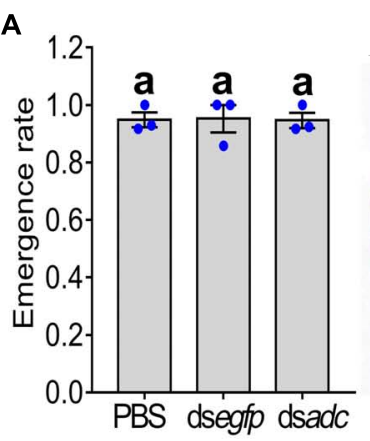

$\mathbf{F}$

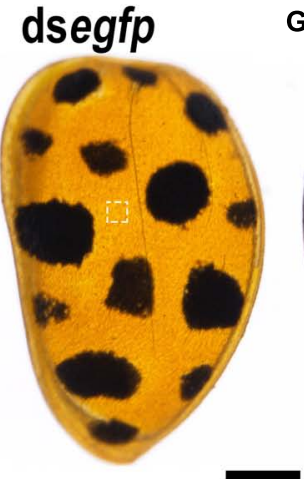

J
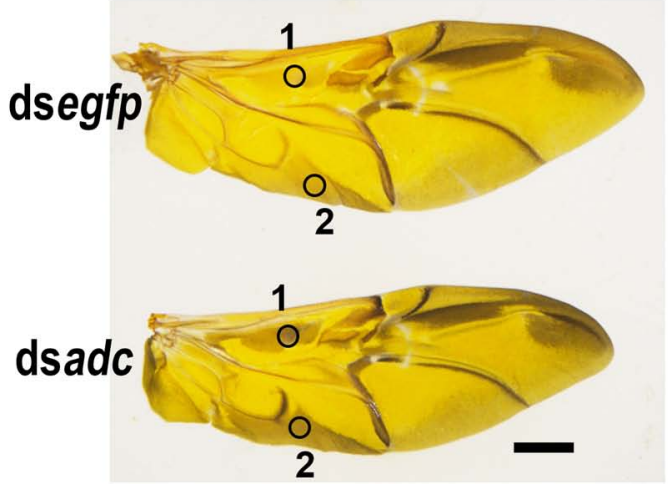

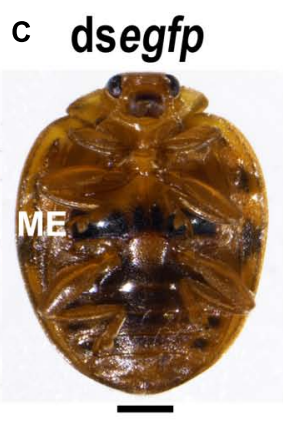

H dsegfp
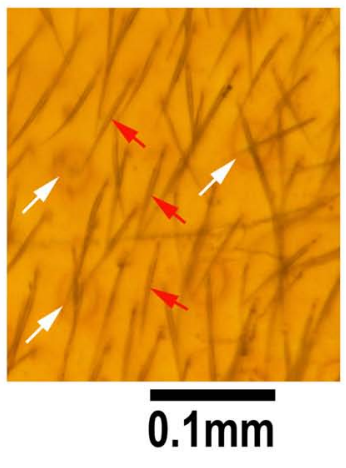

K
D dsadc

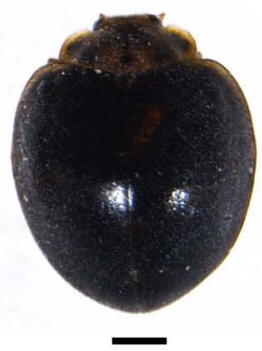

E dsadc

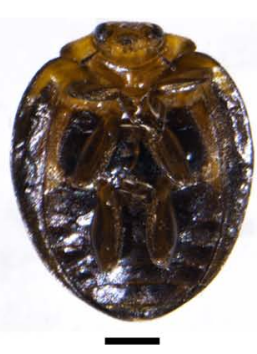

I dsadc

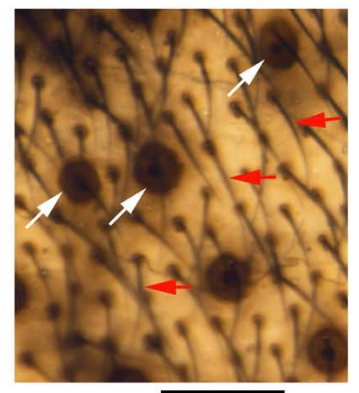

$\overline{0.1 \mathrm{~mm}}$

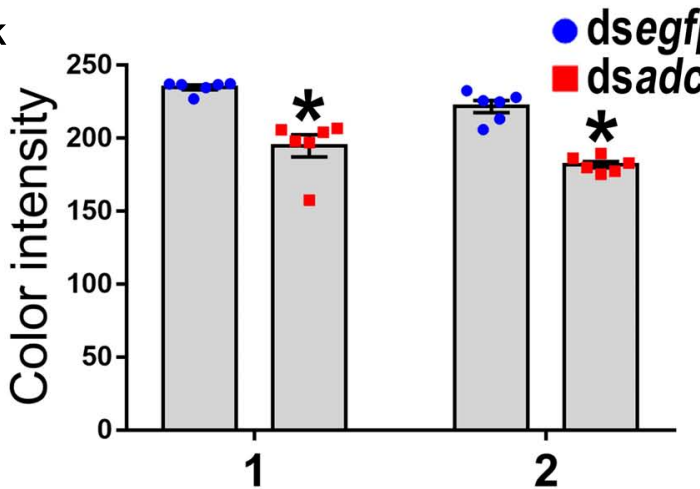

FIGURE 4 | Silencing $H$ vadc affects pigmentation in adults of $H$. vigintioctopunctata. The newly molted third instar larvae were injected with an aliquot (0.1 $\mu$ l) of PBS, or solution including $300 \mathrm{ng}$ dsegfp or $300 \mathrm{ng}$ dsadc. The larvae were then transferred to potato foliage. The emergence rates and the rates of normal adults were recorded during a 3-week trial period (A). The average values $( \pm \mathrm{SE})$ followed by different letters indicate significant differences at $P$-value $<0.05$ using ANOVA with the Tukey-Kramer test. The dorsal $\mathbf{( B , D )}$ and ventral $\mathbf{( C , E ) ~ v i e w s ~ o f ~} 7$-day-old adults whose third instar larvae had been subjected to dsegfp or dsadc treatment are shown. The elytra (F,G) and the elytral surfaces were further amplified $\mathbf{( H , I )}$. The hindwings $\mathbf{( J )}$ and the color intensity $(\mathbf{K})$ in equivalent regions of them (circles 1 and 2 in $\mathbf{J}$ ) were determined as mean gray values (average luminance) by ImageJ software. Data are shown as the mean values \pm SE ( $n=6$ ). The asterisk indicates a significant difference in color intensity between control and test beetles $(P<0.01, t$-test). White and red arrows point to the circle pits and setae, respectively. ME,

\section{Knockdown of Adc Affects the Color of Adult Cuticle and Wings}

The emergence rate of the pupae in the dsadc-treated group was $95 \%$ on average, showing no significant difference with the one in the PBS- or dsegfp-treated group (Figure 4A). For Hvadc RNAi adults, 28 black markings were distributed symmetrically on two hard elytra 1 day after emergence. Then, the black pigments were gradually deposited and finally covered the whole body from the dorsal view 7 days after emergence, while the pigmentation of the control (dsegfp-treated) adult remained the same
(Figures 4B vs. D). Similarly, a pair of black markings on the sternum of the metathorax was seen in the control and 1day-old Hvadc RNAi adults; while the whole body from the ventral view was blackened in the 7-day-old Hvadc RNAi adults (Figures 4C vs. E). Knockdown of Hvadc caused the overall darkening pigmentation in elytra, which seems to be due to the enlargement of the black spots (Figures 4F vs. G). Moreover, the view of the amplified elytra from Hvadc RNAi adults showed that the setae and circle pits were all dark-colored, in contrast to the reddish-brown color in the control beetles (Figures $\mathbf{4 H}$ vs. I). 
Further dissection of the head, pronotum, scutellum, hindlegs, and hindwing revealed that these regions all shifted to the darker pigmentation (Figure 4J and Supplementary Figure 4). The color intensity of the corresponding regions of hindwings showed a significant difference between dsadc and dsegfp treated groups (Figure 4K).

\section{RNA Interference of Ebony on the Third Instar Larvae}

Since both the Adc and Ebony play central roles in the synthesis of the NBAD (Massey et al., 2019), we have knocked down the expression of Hvebony by injection of dsebony to the newly ecdysed third instar larvae (Figure 5A).

The pupation rate and the emergence rate of the Hvebony RNAi larvae were similar to those of the control larvae which were injected PBS or dsegfp (Figures 5B,C). The mandibles were darker-colored in the dsebony-introduced newly molted fourth instar larvae, compared with those in the control larvae treated with PBS or dsegfp (Figures 5D,E, $15 \mathrm{~min}$ ). Eight hours postmolting, the scoli and strumae from the larval thorax to the eighth segment of the abdomen, along with head and leg, became brownish in the PBS- and dsegfp-introduced larvae (Figures 5D,E, above panel). By contrast, the color of the body parts, namely, head, scoli, strumae, and legs became dark brown in the dsebony-treated larvae (Figures 5D,E, lower panels).

Similarly, at the wandering stage, the cuticles of the larvae head capsules, scoli, strumae, legs, and spots on the abdomen were more blackened in Hvebony RNAi larvae than those in the control larvae (Figures 5F,H,J vs. G,I,K).

In the dsebony-introduced pupae, the black dorsal markings in the meso and meta thoraxes and the dark patches in tergite IIV showed no significant difference with PBS or dsegfp-treated pupae (Figure 6A). However, the mandibles were darker in the 3-day/4-day-old Hvebony-silenced pupae, than those in control groups (Figure 6A). Similar to the phenotype of the Hvadc depleted pupae, several small dark spots appeared in the cuticle between two compound eyes of the dsebony-introduced 4-dayold pupae (Figures 6B vs. C). Furthermore, the cuticle of the head, legs, elytra, and pronotum in dsebony-introduced 4day-old pupae became significantly darker than that of pupae injected with PBS or dsegfp (Figures 6B,D,F vs. C,E,G). The enlarged dorsal view displayed that many short black setae and dots appeared on the cuticle of the dsebony-injected 4-day-old pupae to make the cuticle blacker in color than the control (Figures 6F vs. G).

Thirty minutes after emergence, the elytra of the control adults treated with PBS or dsegfp still exhibited light yellow in color. Subsequently, the elytra turned copper yellow, and 28 spots appeared, gradually darkened (Figure 7A, dsegfp-dorsal view). By contrast, knockdown of Hvebony led to an overall gray pigmentation and enlarged 28 dark spots in elytra within 30 min after emergence. The elytra of the Hvebony RNAi adults also gradually darkened (Figure 7A, dsebony-dorsal view), but they looked completely black in color 7 days after emergence (Figures 7B vs. C). Further dissection of the head, pronotum, scutellum, and hindlegs of the Hvebony RNAi adults showed that these regions all turned to darker pigmentation than control adults (Supplementary Figure 4). Similar to the Hvadc-silenced adults, the 28 black spots on the elytra of the Hvebony-silenced adults became larger than those on the control elytra (Figures 7D vs. E), and the color of the setae and pits on the surface of the elytra was darker than that of the control elytra (Figures $7 F$ vs. G). In addition, the pigment regions of the hindwing from the Hvebony RNAi adults were darker than controls (Figures 7H,I).

From the ventral view, six pairs of dark patches appeared along the lateral portions on the abdomen segments of the Hvebony RNAi adults, and the last two pairs extended and merged together. The pale-yellow color outside the dark patches was gradually darkened, until merging the dark patches 2 days after emergence (Figure 7A, dsebony-ventral view). The legs and the abdomen of the Hvebony-depleted adults were blacker in color than the control beetles (Figure 7A, dsebony-ventral view).

\section{RNA Interference of Tan on the Third Instar Larvae}

Two days and three days after injection of dstan to the third instar larvae, the expression of the target Hvtan transcript was significantly reduced, compared with the control larvae injected PBS or dsegfp (Supplementary Figure 5A).

Depletion of Hvtan exerted little influence on pupation and emergence rates (Supplementary Figure 7). The results of color intensity measurement (data not shown) indicated that knockdown of Hvtan had a very limited influence on the cuticle pigmentation at the larval, pupal, and adult stages, compared with the controls subjected to PBS or dsegfp injection (Supplementary Figures 5B,C, 6A-M). In addition, there was no significant difference in the color intensity of the hindwings between dsegfp- and dstan-treated ladybirds (Supplementary Figure 6N).

\section{DISCUSSION}

The conjugation and cross-linking of cuticle proteins during cuticle tanning lead to an insoluble, hard, and darkened redbrown exoskeleton in Coleoptera (Roseland et al., 1987). NBAD, a sclerotic reagent, and a pigment precursor play critical roles in cuticle pigmentation and hardening (Kramer et al., 1984). In this article, three genes (Hvadc, Hvebony, and Hvtan) reported to be involved in NBAD biosynthesis were identified and their role in the pigmentation of cuticle on the larvae, pupae, and adults of $H$. vigintioctopunctata was explored by RNAi in the newly ecdysed third instar larvae. Three pieces of experimental evidence suggested that NBAD plays more important roles in the pigmentation in adults than that in the larvae and pupae of H. vigintioctopunctata.

First, we found that Hvadc, Hvebony, and Hvtan were detected from the embryo (egg) to adults. Moreover, the transcription levels of Hvadc, Hvebony, and Hvtan were high just before and/or right after the molt at the first, second, third, and fourth larval instar and pupal stages (Figure 1). In S. litura, Slebony was expressed at all developing stages, namely, egg, the first through sixth larval instar larvae, prepupa, pupa, and adult (Bi et al., 2019). Similarly, ebony is abundantly expressed at the larval, pupal, and adult stages in Papilio xuthus and 
A

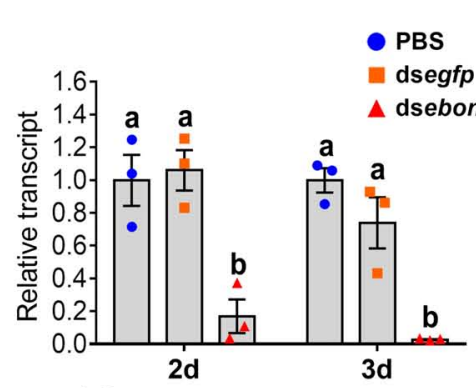

B

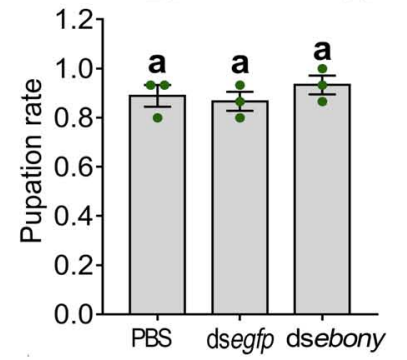

C

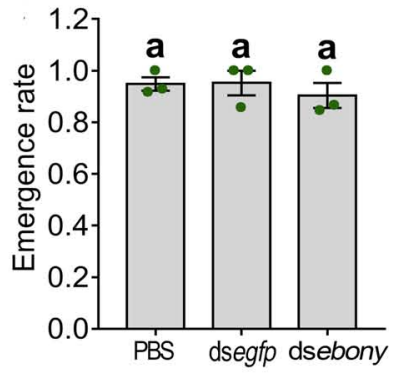

F dsegfp G dsebony
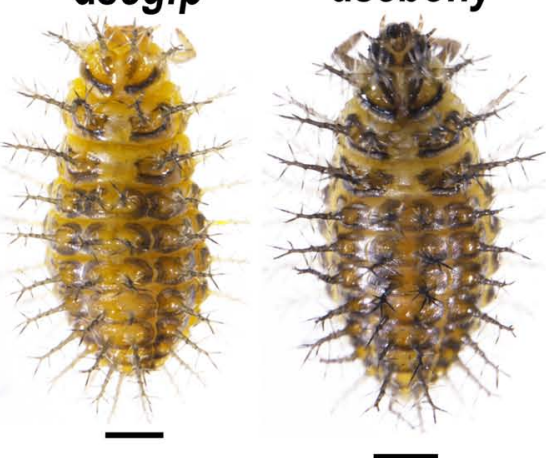

D

\begin{tabular}{|c|c|c|c|c|c|}
\hline \multicolumn{2}{|c|}{$15 \mathrm{~min}$} & \multicolumn{2}{|c|}{$3 h$} & \multicolumn{2}{|c|}{$8 h$} \\
\hline Dorsal & Ventral & Dorsal & Ventral & Dorsal & Ventral \\
\hline
\end{tabular}

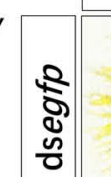

Dorsal
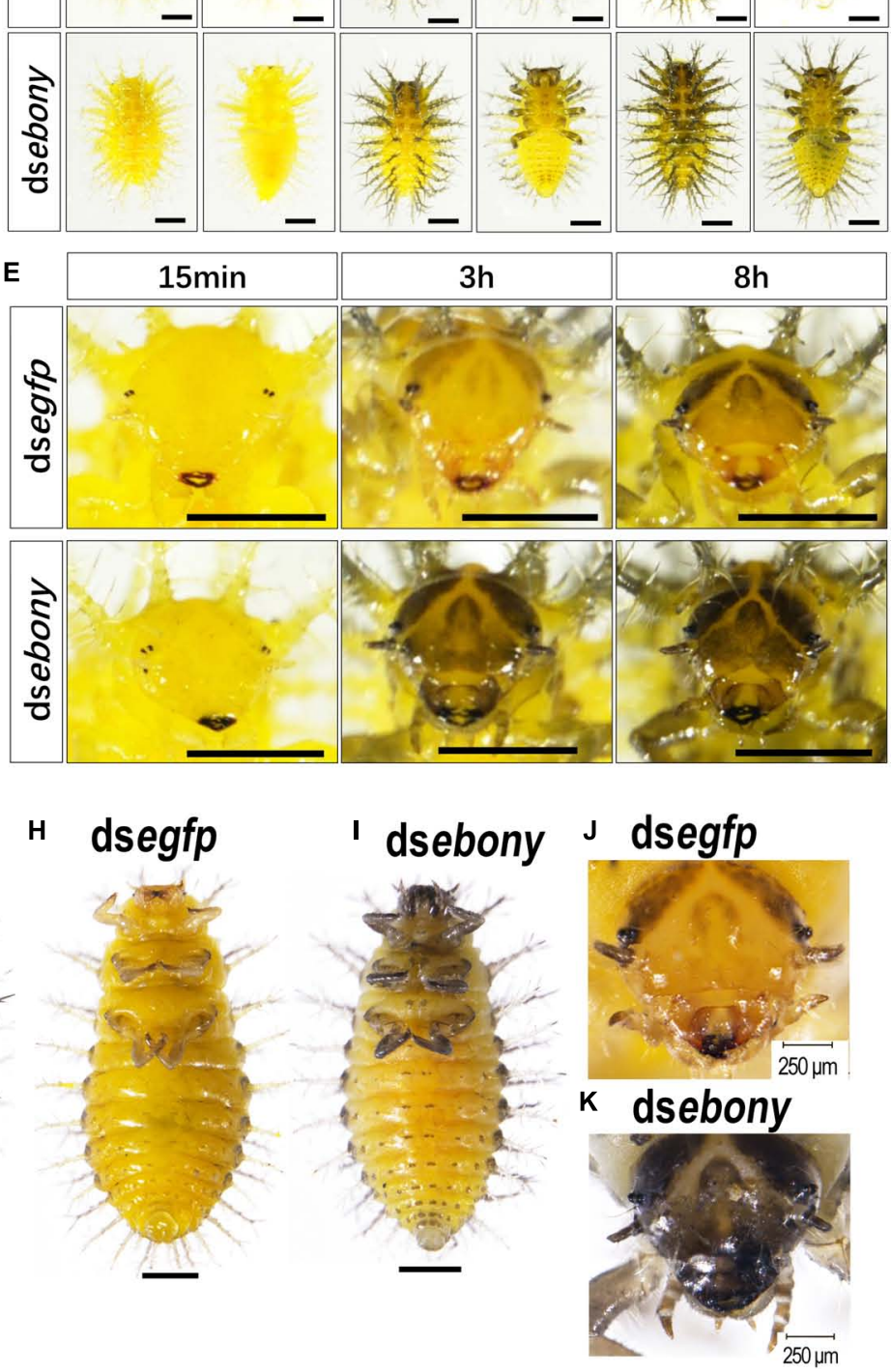

FIGURE 5 | Depletion of Hvebony influences pigmentation in larvae of $H$. vigintioctopunctata. The newly molted third instar larvae were injected with an aliquot $(0.1 \mu l)$ of PBS, or solution including $300 \mathrm{ng}$ dsegfp or $300 \mathrm{ng}$ dsebony. The larvae were then transferred to potato foliage. Two days and three days after injection, the expression level of Hvebony was measured (A). Relative transcripts are the ratios of relative copy numbers in treated individuals to that of PBS-treated ones, which is set as 1. The pupation and emergence rates were recorded during a 3-week trial period $\mathbf{( B , C )}$. Different letters indicate significant difference at $P$-value $<0.05$ using ANOVA with the Tukey-Kramer test. The dorsal and ventral views of dsegfp-and dsebony-treated larvae (D) and amplified larval heads (E) at different times after molting to the fourth instar, and the dorsal and ventral views of larvae in the wandering stage (F-I) are shown. The heads of the wandering stage larvae were further amplified $\mathbf{( J , K )}$. The unlabeled scale bars: $1 \mathrm{~mm}$.

Papilio machaon (Li et al., 2015). In T. molitor, the abundant transcripts were confirmed at late stages of development from pharate pupae through 10-day-old adults (Mun et al., 2020). The expression profiles of Hvadc, Hvebony, and Hvtan suggest that the three functional enzymes catalyze the formation of melanin and sclerotins after each molting during the developing stages.
Second, the expression of the Hvadc, Hvebony, and Hvtan peaked in the 4-day-old pupae or 0-day-old H. vigintioctopunctata adults (Figure 1). The highest expression levels at the late pupal and early adult phrases suggest that abundant NBAD were produced in the preadults and adults. In agreement with these results, the highest level of Tmadc is seen 

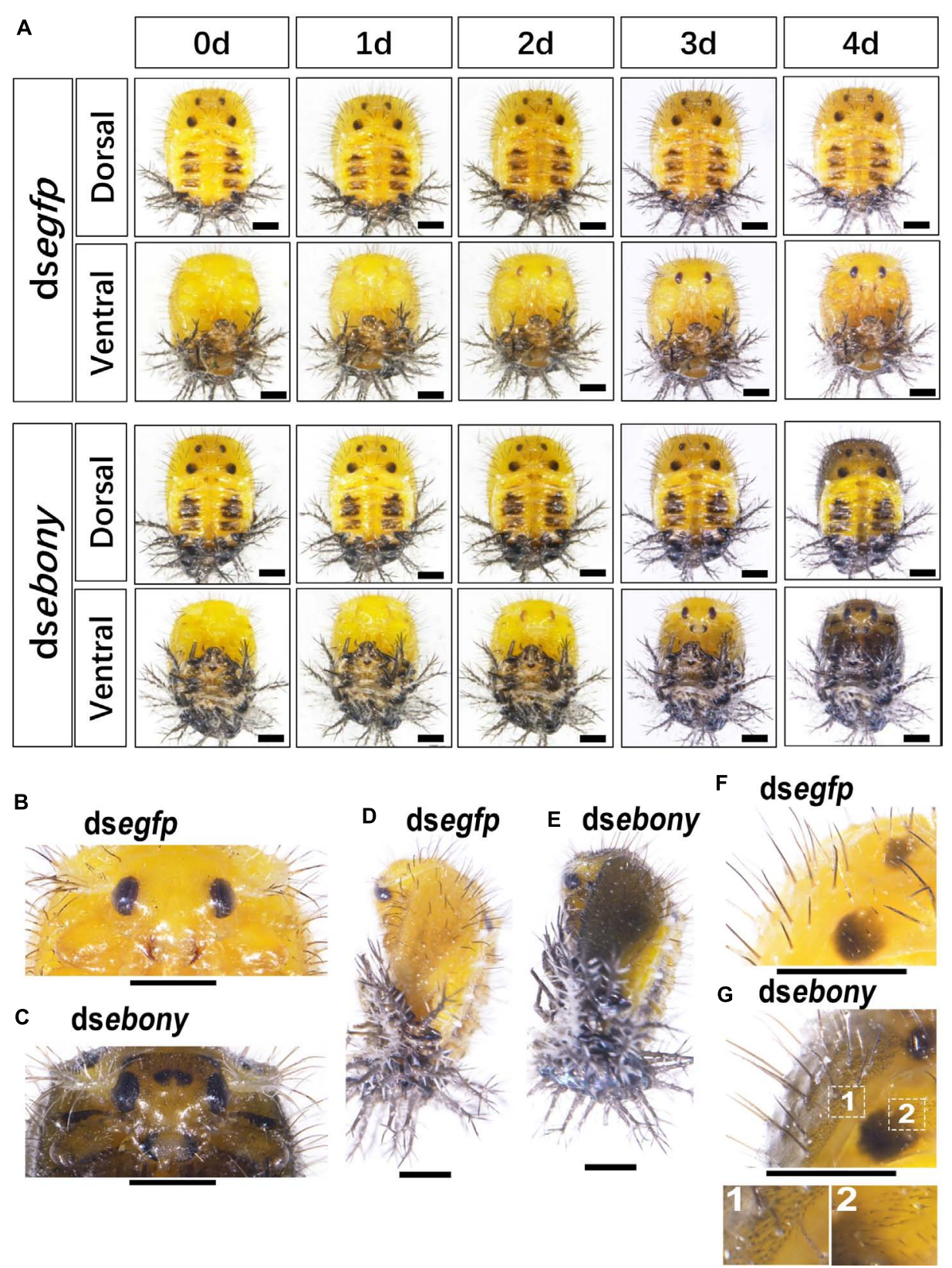

FIGURE 6 | RNA interference of Hvebony affects the pigmentation in pupae of $H$. vigintioctopunctata. The dorsal and ventral views of 0-day, 1-day, 2-day, 3-day, and 4-day old pupae from the third instar larvae injected with dsegfp and dsebony $\mathbf{( A )}$ are shown. The heads $\mathbf{( B , C )}$ and the dorsal view $\mathbf{( F , G ) ~ o f ~ t h e ~ 4 - d a y - o l d ~ p u p a e ~}$ were further amplified. The lateral views of 4-day-old pupae (D,E) are shown. The unlabeled scale bars: $1 \mathrm{~mm}$.

right after adult emergence (adult day 0) in T. molitor (Mun et al., 2020). Similarly, high levels of Slebony were found just before and right after adult emergence in S. litura (Bi et al., 2019). Moreover, Dmadc was expressed at high levels at the end of the pupae period (96 $\mathrm{h}$ after pupation) in $D$. melanogaster (Sobala and Adler, 2016).

Lastly, the results revealed that RNAi of either Hvadc or Hvebony selectively deepened the color of the larval head capsules, scoli, strumae, and legs (Figures 2, 5), and depletion of Hvadc or Hvebony blackened the pupal head capsules, antennae, mouthpart, and legs (Figures 3, 6), while knockdown of either Hvadc or Hvebony darkened whole bodies of the adults (Figures 4,7 ) of $H$. vigintioctopunctata. The greater accumulation of black pigments in the Hvadc or Hvebony depleted adults likely resulted from the conversion of larger quantities of dopamine to brown dopamine-melanin than in the larvae. Therefore, we infer that larger quantities of NBAD were present in adults than larvae/pupae. 


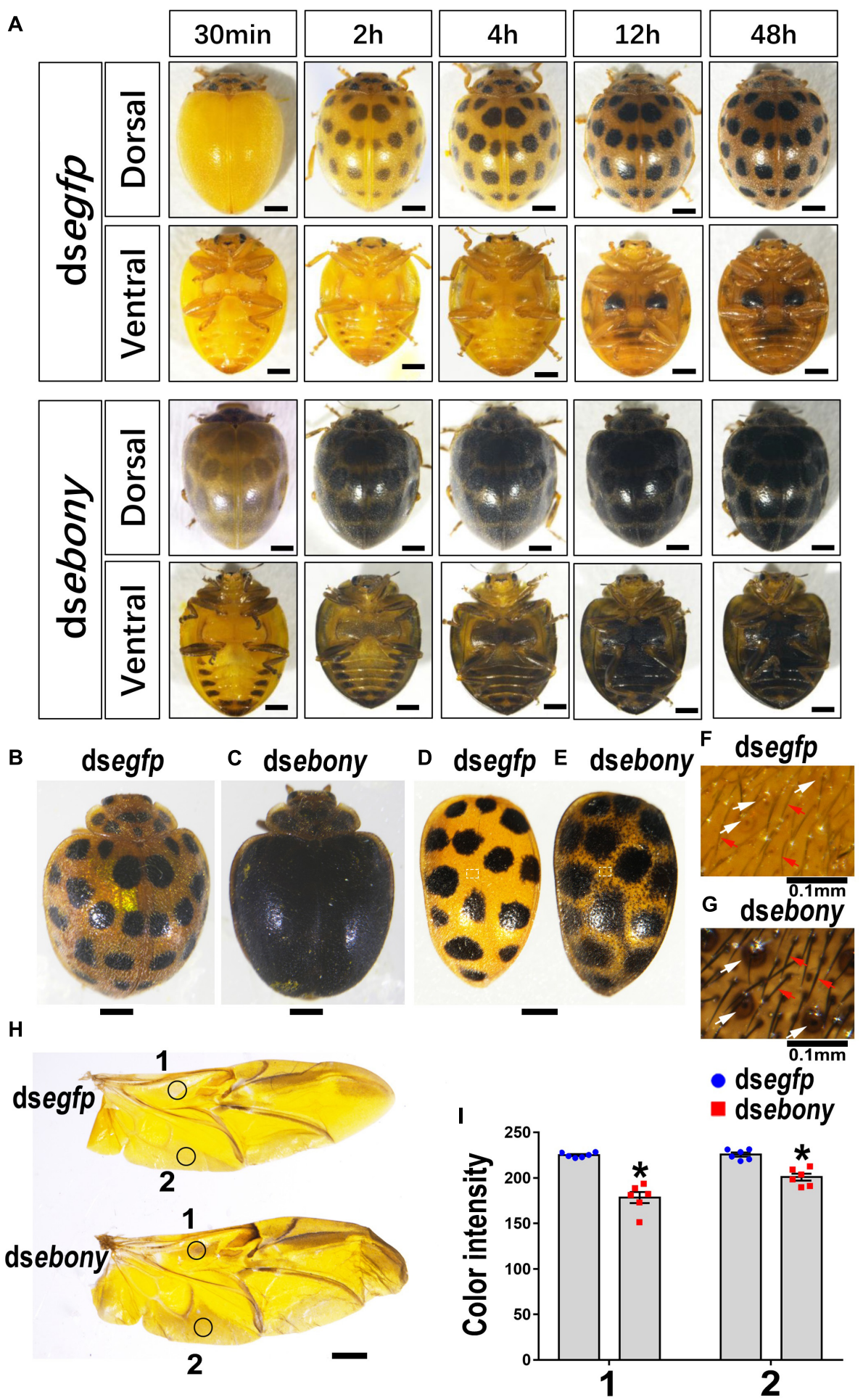

FIGURE 7 | Depletion of Hvebony deepens color of adults in $\mathrm{H}$. vigintioctopunctata. The dorsal and ventral views of adults from different times after emergence (A). The 7-day-old adults (B,C) and elytra dissected from 7-day-old adults (D,E) are shown. The surfaces of elytra were further amplified (F,G). The hindwings $\mathbf{( H )}$ and the color intensity (I) in equivalent regions of them (circles 1 and 2 in $\mathbf{H}$ ) were determined as mean gray values (average luminance) by Image $\mathbf{J}$ software. Data are shown as the mean values \pm SE $(n=6)$. The asterisk indicates a significant difference in color intensity between control and test beetles $(P<0.01, t$-test). The unlabeled scale bars: $1 \mathrm{~mm}$. White and red arrows point to the pits and setae, respectively. 
In contrast, microinjection of dsadc into penultimate-instar larvae does not cause obvious phenotype in the $T$. castaneum old larvae but leads to a darkened body pigmentation in pupae and adults when compared with controls (Arakane et al., 2009). In the Bmadc mutant strain of B. mori, $\beta$-alanine and NBAD were deficient but dopamine is accumulated. As a result, deep coloration was induced in pupae (Dai et al., 2015). It appears that NBAD is as important in pupae as that in adults in both T. castaneum (Arakane et al., 2009) and B. mori (Dai et al., 2015). As for ebony, CRISPR/Cas9-mediated ebony knockout did not change the pigmentation in larvae but led to darker coloration in the pupae and adults in S. litura (Bi et al., 2019). In D. melanogaster, homozygous mutations of ebony bring about dark body pigmentation in adult flies, but not larvae and pupae (Massey et al., 2019). Moreover, the core enhancers in the ebony gene determine thoracic pigmentation intensity in different D. melanogaster strains across geographical gradients (TelonisScott et al., 2011; Telonis-Scott and Hoffmann, 2018). These results suggest that reduced synthesis of NBAD leads to abnormal accumulations of high levels of dopamine, which likely undergoes dopamine-melanin synthesis to cause a dark coloration of cuticle in adults of insects from different orders. Interestingly, although the elytra of adults treated with dsadc and dsebony appear to be almost completely black in color, we found that the main regions of elytra got dark pigmented were pits and setae (Figures 4, 7). Similarly, as early as the end of the pupae, the epidermal setae were already black in coloration in the Hvebony depleted pupae (Figure 6). It suggested that NBAD may be mainly accumulated in pits and setae of $H$. vigintioctopunctata cuticle.

It is known that NBAD is a major sclerotizing precursor that is secreted during the cuticle tanning process. It is further oxidized and used to cross-link cuticular proteins to form a rigid coleopteran exoskeleton at the adult stage (Noh et al., 2016). Moreover, some pigmentation gene transcripts, such as ebony and tan, are pleiotropic genes that affect more than one trait. In D. melanogaster, loss-of-function mutations in ebony and tan also affect cuticular hydrocarbon composition (Massey et al., 2019). A bias for long-chain hydrocarbon increases the melting temperatures and enables the flies to live longer in dry climates (Gibbs, 1998). H. vigintioctopunctata is a diurnal herbivorous ladybird. The adults are frequently exposed to a dry environment to forage for food, oviposition sites, and copulation mates. Hard exoskeleton in the adults confers greater tolerance to desiccation and ultraviolet during the foraging stage (Matute and Harris, 2013; Bastide et al., 2014).

In addition, we noticed that the change of cuticle pigmentation was very limited in the beetles treated with dstan (Supplementary Figures 5, 6). The enzyme encoded by tan is required for the production of dopamine in D. melanogaster (True et al., 2005). In B. mori, tan is suggested to be the responsible gene for larval color mutant rouge, and Tan plays a significant role

\section{REFERENCES}

Andersen, S. O. (2010). Insect cuticular sclerotization: a review. Insect Biochem. Mol. Biol. 40, 166-178. doi: 10.1016/j.ibmb.2009.10.007 in emphasizing the black markings of the larvae (Futahashi et al., 2010). Previous studies have shown that Tan catalyzes the production of dopamine from NBAD during pigment development (True et al., 2005). This role of Tan seems to be not obvious in $H$. vigintioctopunctata. Combined with the results of RNAi of Hvadc and Hvebony, we speculated that Hvtan plays a weak role in the process of converting NBAD to dopamine in $H$. vigintioctopunctata.

\section{CONCLUSION}

In conclusion, the study has determined the important role of Adc and Ebony in cuticle pigmentation in the 28-spotted ladybird. Larger quantities of NBAD were suggested to be present in adults and play more important roles in pigmentation than larvae/pupae. Abundant NBAD in the adults may be an adaptation strategy in the tanning process to form inconspicuous pigmentation in $H$. vigintioctopunctata beetles.

\section{DATA AVAILABILITY STATEMENT}

The datasets presented in this study can be found in online repositories. The names of the repository/repositories and accession number(s) can be found in the article/Supplementary Material.

\section{AUTHOR CONTRIBUTIONS}

L-JZ, LJ, and G-QL conceived the study, participated in the design of the experiments and the interpretation of the results, and wrote the first draft of the manuscript. L-JZ and LJ performed the experiments. LJ and G-QL revised the manuscript. All authors contributed to the article and approved the submitted version of the manuscript.

\section{FUNDING}

This study was supported by the National Natural Science Foundation of China (32072416) and the China Agriculture Research System of MOF and MARA (CARS-09-P22).

\section{SUPPLEMENTARY MATERIAL}

The Supplementary Material for this article can be found online at: https://www.frontiersin.org/articles/10.3389/fphys. 2022.829675/full\#supplementary-material

Arakane, Y., Lomakin, J., Beeman, R. W., Muthukrishnan, S., Gehrke, S. H., Kanost, M. R., et al. (2009). Molecular and functional analyses of amino acid decarboxylases involved in cuticle tanning in Tribolium castaneum. J. Biol. Chem. 284, 16584-16594. doi: 10.1074/jbc.M901629200 
Bastide, H., Yassin, A., Johanning, E. J., and Pool, J. E. (2014). Pigmentation in Drosophila melanogaster reaches its maximum in Ethiopia and correlates most strongly with ultra-violet radiation in sub-Saharan Africa. BMC Evol. Biol. 14:179. doi: 10.1186/s12862-014-0179-y

Bi, H. L., Xu, J., He, L., Zhang, Y., Li, K., and Huang, Y. P. (2019). CRISPR/Cas9mediated ebony knockout results in puparium melanism in Spodoptera litura. Insect Sci. 26, 1011-1019. doi: 10.1111/1744-7917.12663

Borycz, J., Borycz, J. A., Edwards, T. N., Boulianne, G. L., and Meinertzhagen, I. A. (2012). The metabolism of histamine in the Drosophila optic lobe involves an ommatidial pathway: $\beta$-alanine recycles through the retina. J. Exp. Biol. 215, 1399-1411. doi: 10.1242/jeb.060699

Bridges, C. B., and Morgan, T. H. (1923). The Third-Chromosome Group of Mutant Characters of Drosophila melanogaster. Washington, DC: Carnegie Institution of Washington.

Bustin, S. A., Benes, V., Garson, J. A., Hellemans, J., Huggett, J., Kubista, M., et al. (2009). The MIQE guidelines: minimum information for publication of quantitative real-time PCR experiments. Clin. Chem. 55, 611-622. doi: 10.1373/ clinchem.2008.112797

Casari, S. A., and Teixeira, E. P. (2015). Immatures of epilachna chevrolat (Coleoptera, Coccinellidae, Epilachninae). Rev. Bras. Entomol. 59, 113-120. doi: 10.1016/j.rbe.2015.03.006

Dai, F. Y., Qiao, L., Cao, C., Liu, X. F., Tong, X. L., He, S. Z., et al. (2015). Aspartate decarboxylase is required for a normal pupa pigmentation pattern in the silkworm, Bombyx mori. Sci. Rep. 5:10885. doi: 10.1038/srep 10885

Ferguson, L. C., Maroja, L., and Jiggins, C. D. (2011). Convergent, modular expression of ebony and tan in the mimetic wing patterns of Heliconius butterflies. Dev. Genes Evol. 221, 297-308. doi: 10.1007/s00427-011-0380-6

Futahashi, R., Banno, Y., and Fujiwara, H. (2010). Caterpillar color patterns are determined by a two-phase melanin gene prepatterning process: new evidence from tan and laccase2. Evol. Dev. 12, 157-167. doi: 10.1111/j.1525-142X.2010. 00401.x

Futahashi, R., Sato, J., Meng, Y., Okamoto, S., Daimon, T., Yamamoto, K., et al. (2008). yellow and ebony are the responsible genes for the larval color mutants of the silkworm Bombyx Mori. Genetics 180, 1995-2005. doi: 10.1534/genetics. 108.096388

Gibbs, A. G. (1998). Water-proofing properties of cuticular lipids. Am. Zool. 38, 471-482. doi: $10.1093 / \mathrm{icb} / 38.3 .471$

Hodgetts, R., and Choi, A. (1974). $\beta$ Alanine and cuticle maturation in Drosophila. Nature 252, 710-711. doi: 10.1038/252710a0

Jacobs, M. E. (1974). Beta-alanine and adaptation in Drosophila. J. Insect Physiol. 20, 859-866. doi: 10.1016/0022-1910(74)90175-9

Jeong, S., Rebeiz, M., Andolfatto, P., Werner, T., True, J., and Carroll, S. B. (2008). The evolution of gene regulation underlies a morphological difference between two Drosophila sister species. Cell 132, 783-793. doi: 10.1016/j.cell.2008. 01.014

Kramer, K. J., Morgan, T. D., Hopkins, T. L., Roseland, C. R., Aso, Y., Beeman, R. W., et al. (1984). Catecholamines and $\beta$-alanine in the red flour beetle, Tribolium castaneum: roles in cuticle sclerotization and melanization. Insect Biochem. 14, 293-298. doi: 10.1016/0020-1790(84)90063-5

Li, X. Y., Fan, D. D., Zhang, W., Liu, G. C., Zhang, L., Zhao, L., et al. (2015). Outbred genome sequencing and CRISPR/Cas9 gene editing in butterflies. Nat. Commun. 6:8212. doi: 10.1038/Ncomms 9212

Liu, J., Lemonds, T. R., Marden, J. H., and Popadic, A. (2016). A pathway analysis of melanin patterning in a Hemimetabolous insect. Genetics 203:403. doi: 10.1534/ genetics.115.186684

Lü, J., Chen, S. M., Guo, M. J., Ye, C. Y., Qiu, B. L., Wu, J. H., et al. (2018). Selection and validation of reference genes for RT-qPCR analysis of the ladybird beetle Henosepilachna vigintioctomaculata. Front. Physiol. 9:1614. doi: 10.3389/Fphys. 2018.01614

Massey, J. H., Akiyama, N., Bien, T., Dreisewerd, K., Wittkopp, P. J., Yew, J. Y., et al. (2019). Pleiotropic effects of ebony and tan on pigmentation and cuticular hydrocarbon composition in Drosophila melanogaster. Front. Physiol. 10:518. doi: 10.3389/Fphys.2019.00518

Matute, D. R., and Harris, A. (2013). The influence of abdominal pigmentation on desiccation and ultraviolet resistance in two species of Drosophila. Evolution 67, 2451-2460. doi: 10.1111/evo. 12122

Mun, S., Noh, M. Y., Kramer, K. J., Muthukrishnan, S., and Arakane, Y. (2020). Gene functions in adult cuticle pigmentation of the yellow mealworm, Tenebrio molitor. Insect Biochem. Mol. Biol. 117:103291. doi: 10.1016/J.Ibmb.2019. 103291

Noh, M. Y., Koo, B., Kramer, K. J., Muthukrishnan, S., and Arakane, Y. (2016). Arylalkylamine $\mathrm{N}$-acetyltransferase 1 gene (TcAANAT1) is required for cuticle morphology and pigmentation of the adult red flour beetle, Tribolium castaneum. Insect Biochem. Mol. Biol. 79, 119-129. doi: 10.1016/j.ibmb.2016.10. 013

Phillips, A. M., Smart, R., Strauss, R., Brembs, B., and Kelly, L. E. (2005). The Drosophila black enigma: the molecular characterization of the black1 mutant and behavioural allele. Gene 351, 131-142. doi: 10.1016/j.gene.2005.03.013

Roseland, C. R., Kramer, K. J., and Hopkins, T. L. (1987). Cuticular strength and pigmentation of rust-red and black strains of Tribolium castaneum: correlation with catecholamine and $\beta$-alanine content. Insect Biochem. 17, 21-28. doi: 10. 1016/0020-1790(87)90139-9

Simon, J. D., Peles, D., Wakamatsu, K., and Ito, S. (2009). Current challenges in understanding melanogenesis: bridging chemistry, biological control, morphology, and function. Pigm. Cell Melanoma R. 22, 563-579. doi: 10.1111/ j.1755-148X.2009.00610.x

Sobala, L. F., and Adler, P. N. (2016). The gene expression program for the formation of wing cuticle in Drosophila. PLoS Genet. 12:e1006100. doi: 10.1371/ journal.pgen.1006100

Sugumaran, M., and Barek, H. (2016). Critical analysis of the melanogenic pathway in insects and higher animals. Int. J. Mol. Sci. 17:1753. doi: 10.3390/ Ijms17101753

Telonis-Scott, M., and Hoffmann, A. A. (2018). Enhancing ebony? common associations with a cis-regulatory haplotype for Drosophila melanogaster thoracic pigmentation in a Japanese population and Australian populations. Front. Physiol. 9:822. doi: 10.3389/Fphys.2018. 00822

Telonis-Scott, M., Hoffmann, A. A., and Sgro, C. M. (2011). The molecular genetics of clinal variation: a case study of ebony and thoracic trident pigmentation in Drosophila melanogaster from eastern Australia. Mol. Ecol. 20, 2100-2110. doi: 10.1111/j.1365-294X.2011.05 089.x

Tomoyasu, Y., Arakane, Y., Kramer, K. J., and Denell, R. E. (2009). Repeated co-options of exoskeleton formation during wing-to-elytron evolution in beetles. Curr. Biol. 19, 2057-2065. doi: 10.1016/j.cub.2009. 11.014

True, J. R. (2003). Insect melanism: the molecules matter. Trends Ecol. Evol. 18, 640-647. doi: 10.1016/j.tree.2003.09.006

True, J. R., Yeh, S. D., Hovemann, B. T., Kemme, T., Meinertzhagen, I. A., Edwards, T. N., et al. (2005). Drosophila tan encodes a novel hydrolase required in pigmentation and vision. PLoS Genet. 1:e63. doi: 10.1371/journal.pgen.001 0063

van't Hof, A. E., Edmonds, N., Dalikova, M., Marec, F., and Saccheri, I. J. (2011). Industrial melanism in British peppered moths has a singular and recent mutational origin. Science 332, 958-960. doi: 10.1126/science.120 3043

Wappner, P., Hopkins, T. L., Kramer, K. J., Cladera, J. L., Manso, F., and QuesadaAllue, L. A. (1996a). Role of catecholamines and $\beta$-alanine in puparial color of wild-type and melanic mutants of the Mediterranean fruit fly (Ceratitis capitata). J. Insect Physiol. 42, 455-461. doi: 10.1016/0022-1910(95)0 0131-X

Wappner, P., Kramer, K. J., Manso, F., Hopkins, T. L., and QuesadaAllue, L. A. (1996b). N- $\beta$-alanyldopamine metabolism for puparial tanning in wild-type and mutant Niger strains of the Mediterranean fruit fly, Ceratitis capitata. Insect Biochem. Mol. Biol. 26, 585-592. doi: 10.1016/S0965-1748(96)00012-4

Wittkopp, P. J., and Beldade, P. (2009). Development and evolution of insect pigmentation: genetic mechanisms and the potential consequences of pleiotropy. Semin. Cell Dev. Biol. 20, 65-71. doi: 10.1016/j.semcdb.2008.10.002

Wittkopp, P. J., Carroll, S. B., and Kopp, A. (2003). Evolution in black and white: genetic control of pigment patterns in Drosophila. Trends Genet. 19, 495-504. doi: 10.1016/S0168-9525(03)00194-X 
Wright, T. R. F. (1987). The genetics of biogenic amine metabolism, sclerotization, and melanization in Drosophila melanogaster. Adv. Genet. 24, 127-222. doi: 10.1016/s0065-2660(08)60008-5

Xu, P., Ze, L. J., Kang, W. N., Wu, J. J., Jin, L., Anjum, A. A., et al. (2020). Functional divergence of white genes in Henosepilachna vigintioctopunctata revealed by RNA interference. Insect Mol. Biol. 29, 466-476. doi: 10.1111/imb.12656

Ze, L. J., Xu, P., Kang, W. N., Wu, J. J., Jin, L., Anjum, A. A., et al. (2021). Disruption of kynurenine pathway reveals physiological importance of tryptophan catabolism in Henosepilachna vigintioctopunctata. Amino Acids 53, 1091-1104. doi: 10.1007/s00726-021-03009-4

Zhan, S., Guo, Q., Li, M., Li, M., Li, J., Miao, X., et al. (2010). Disruption of an $\mathrm{N}$-acetyltransferase gene in the silkworm reveals a novel role in pigmentation. Development 137, 4083-4090. doi: 10.1242/dev.053678

Zhang, Q. L., Wang, F., Guo, J., Deng, X. Y., Chen, J. Y., and Lin, L. B. (2018). Characterization of ladybird Henosepilachna vigintioctopunctata transcriptomes across various life stages. Sci. Data 5:180093. doi: 10.1038/Sdata. 2018.93

Ziegler, A. B., Brusselbach, F., and Hovemann, B. T. (2013). Activity and coexpression of Drosophila black with ebony in fly optic lobes reveals putative cooperative tasks in vision that evade electroretinographic detection. J. Comp Neurol. 521, 1207-1224. doi: 10.1002/cne.23247

Conflict of Interest: The authors declare that the research was conducted in the absence of any commercial or financial relationships that could be construed as a potential conflict of interest.

Publisher's Note: All claims expressed in this article are solely those of the authors and do not necessarily represent those of their affiliated organizations, or those of the publisher, the editors and the reviewers. Any product that may be evaluated in this article, or claim that may be made by its manufacturer, is not guaranteed or endorsed by the publisher.

Copyright (c) $2022 \mathrm{Ze}$, Jin and Li. This is an open-access article distributed under the terms of the Creative Commons Attribution License (CC BY). The use, distribution or reproduction in other forums is permitted, provided the original author(s) and the copyright owner(s) are credited and that the original publication in this journal is cited, in accordance with accepted academic practice. No use, distribution or reproduction is permitted which does not comply with these terms. 